\title{
Investigation of a Modified Mid-Point Quadrature Formula
}

\author{
By D. Jagerman
}

1. Introduction. The mid-point quadrature formula

$$
\int_{0}^{1} f(x) d x \cong \frac{1}{N} \sum_{j=1}^{N} f\left(\frac{2 j-1}{2 N}\right)
$$

possesses the valuable attribute that its coefficients are equal. This provides a convenience in computation; further, if the quantities $f((2 j-1) / 2 N)$ are measured, and hence subject to random error, then, on a minimum variance basis and the requirement that the quadrature be exact for $f(x) \equiv 1$, the coefficients of the best linear quadrature formula are equal. In some applications, equal coefficients may be especially important. This arises, for example, in certain designs of phased array antennas where engineering constraints imply the requirement of equal coefficients.

Let a weight function $p(x)$ satisfy

$$
p(x) \geqq 0, \quad \int_{0}^{1} p(x) d x=1,
$$

then it is desirable to obtain an equal-coefficient formula for

$$
I(f)=\int_{0}^{1} p(x) f(x) d x .
$$

The function

$$
L(x)=\int_{0}^{x} p(u) d u
$$

satisfies

$$
L(0)=0, \quad L(1)=1
$$

and is monotonic increasing. Let

$$
y=L(x), \quad x=G(y)
$$

in which $G(y)$ is the function inverse to $L(x)$, then, defining the numbers $x_{1}$, $x_{2}, \cdots, x_{N}$ by

$$
x_{j}=G\left(\frac{2 j-1}{2 N}\right), \quad 1 \leqq j \leqq N,
$$

the required quadrature formula is given by

$$
S_{N}(f)=\frac{1}{N} \sum_{j=1}^{N} f\left(x_{j}\right) .
$$

Received July 13, 1965. 
The sum $S_{N}(f)$ is clearly a Riemannian sum for

$$
\bar{I}(f)=\int_{0}^{1} f[G(y)] d y
$$

and hence, if $f(x)$ is Riemann integrable,

$$
\lim _{N \rightarrow \infty} S_{N}(f)=\bar{I}(f) ;
$$

however, using (1.6) and (1.3),

$$
\bar{I}(f)=I(f)
$$

and hence the approximation is secured.

In order to use the quadrature sum (1.8) effectively, it is necessary to have accurate estimates for the error $R_{N}(f)$ defined by

$$
R_{N}(f)=I(f)-S_{N}(f) .
$$

The class of functions for which $R_{N}(f)$ will be studied are those whose first derivative is of bounded variation over $[0,1]$ and periodically extended with period one. At a point of discontinuity $c$, the value $f(c)$ will be defined by

$$
f(c)=\frac{f(c+)+f(c-)}{2},
$$

in which the plus and minus signs indicate limit from the right and limit from the left respectively.

Section 2 of the paper introduces and proves three theorems for the estimation of $R_{N}(f)$. Theorem 1 provides an estimate of $\left|R_{N}(f)\right|$ in terms of $f^{\prime}(1)$ and the variation $\mathrm{V}_{0}^{1}\left(f^{\prime}\right)$ of $f^{\prime}(x)$ over $[0,1]$. The essential feature is the existence of some order of derivative, say the $r$ th, of $L(x)$ which is bounded away from zero; then

$$
R_{N}(f)=O\left(N^{-1-1 / r}\right) .
$$

The class of Stieltjes bandlimited functions, that is functions for which there exist a constant $\sigma>0$ and a function $\alpha(u) \in \mathrm{BV}[-\sigma, \sigma]$ so that

$$
f(x)=\int_{-\sigma}^{\sigma} e^{i u x} d \alpha(u)
$$

is an important subclass of $f^{\prime}(x) \in \mathrm{BV}[0,1]$. By utilizing the representation (1.15), Theorem 2 provides an estimate for $\left|R_{N}(f)\right|$ which may be stronger than that provided by Theorem 1. The improvement lies in the constant of the 0 in (1.14).

The special case $p(x) \equiv 1$ is not covered by Theorems 1 and 2 ; however, Theorem 3 provides the sharp estimate

$$
\left|R_{N}(f)\right| \leqq \frac{\mathrm{V}_{0}^{1}\left(f^{\prime}\right)}{16 N^{2}} .
$$

In Section 3 an application of the quadrature theory is made to the representation of a function $H(t)(-\infty<t<\infty)$ by means of an equal-weighted average of some given function $f(t, x)$ over the values $f\left(t, x_{j}\right)$. 
2. Analytical Discussion. In order to establish the main theorems, the following two lemmas will be needed.

LEMMA 1. $c_{n}=\int_{0}^{1} f^{\prime}(x) \sin [2 \pi n L(x)] d x$

$$
\Rightarrow R_{N}(f)=\frac{1}{\pi N} \sum_{k=1}^{\infty}(-1)^{k} \frac{c_{N k}}{k} .
$$

Proof. It will be convenient to introduce the function

$$
S_{N}(f ; y)=\frac{1}{N} \sum_{j=1}^{N} f\left[G\left(y+\frac{2 j-1}{2 N}\right)\right] ;
$$

thus

$$
S_{N}(f ; 0)=S_{N}(f) .
$$

The function $f[G(y)]$ may be expanded into a Fourier series on the interval $(0,1)$; one has

$$
f[G(y)] \stackrel{\doteq}{=}(f)+\sum_{n=1}^{\infty} a_{n} \cos 2 \pi n y+\sum_{n=1}^{\infty} b_{n} \sin 2 \pi n y,
$$

in which

$$
\begin{aligned}
& a_{n}=2 \int_{0}^{1} f[G(y)] \cos 2 \pi n y d y \\
& b_{n}=2 \int_{0}^{1} f[G(y)] \sin 2 \pi n y d y .
\end{aligned}
$$

Define $c_{n}, d_{n}$ by

$$
\begin{aligned}
& c_{n}=\int_{0}^{1} G^{\prime}(y) f^{\prime}[G(y)] \sin 2 \pi n y d y=\int_{0}^{1} f^{\prime}(x) \sin [2 \pi n L(x)] d x, \\
& d_{n}=\int_{0}^{1} G^{\prime}(y) f^{\prime}[G(y)] \cos 2 \pi n y d y=\int_{0}^{1} f^{\prime}(x) \cos [2 \pi n L(x)] d x,
\end{aligned}
$$

then integration by parts applied to (2.4) and (2.5) yields

$$
\begin{aligned}
& a_{n}=-\frac{1}{\pi n} c_{n}, \\
& b_{n}=\frac{f(0+)-f(1-)}{\pi n}+\frac{1}{\pi n} d_{n} .
\end{aligned}
$$

The Bernoullian function

$$
\rho(y)=\frac{1}{2}-\{y\},
$$

in which $\{y\}$ designates the fractional part of $y$, has the Fourier series

$$
\rho(y)=\sum_{n=1}^{\infty} \frac{\sin 2 \pi n y}{\pi n},
$$


hence, replacing $a_{n}, b_{n}$ in (2.3) by their values in (2.8), (2.9), one obtains

$$
\begin{aligned}
f[G(y)]=I(f) & -\frac{1}{\pi} \sum_{n=1}^{\infty} \frac{c_{n}}{n} \cos 2 \pi n y \\
& +\frac{1}{\pi} \sum_{n=1}^{\infty} \frac{d_{n}}{n} \sin 2 \pi n y+[f(0+)-f(1-)] \rho(y) .
\end{aligned}
$$

By summation of a geometric series, one has

$$
\begin{aligned}
\frac{1}{N} \sum_{j=1}^{N} \exp \left(i 2 \pi n\left(y+\frac{2 j-1}{2 N}\right)\right) & =(-1)^{n / N} \exp (i 2 \pi n y), \quad N \mid n, \\
& =0, \quad N \nmid n,
\end{aligned}
$$

and hence, letting $n=N k$ ( $k>0$ integral),

$$
\begin{gathered}
\frac{1}{N} \sum_{j=1}^{N} \cos 2 \pi N k\left(y+\frac{2 j-1}{2 N}\right)=(-1)^{k} \cos 2 \pi N k y, \\
\frac{1}{N} \sum_{j=1}^{N} \sin 2 \pi N k\left(y+\frac{2 j-1}{2 N}\right)=(-1)^{k} \sin 2 \pi N k y .
\end{gathered}
$$

Equations (2.1), (2.12), (2.14), and (2.15) now yield

$$
\begin{aligned}
S_{N}(f ; y)=I(f) & -\frac{1}{\pi N} \sum_{k=1}^{\infty}(-1)^{h} \frac{c_{N k}}{k} \cos 2 \pi N k y \\
& +\frac{1}{\pi N} \sum_{k=1}^{\infty}(-1)^{k} \frac{d_{N k}}{k} \sin 2 \pi N k y \\
& +[f(0+)-f(1-)] \frac{1}{N} \sum_{j=1}^{N} \rho\left(y+\frac{2 j-1}{2 N}\right) .
\end{aligned}
$$

The Fourier series for $\rho(y),(2.11)$, permits ready establishment of the identity

$$
\sum_{j=1}^{N} \rho\left(y+\frac{2 j-1}{2 N}\right)=\rho\left(N y+\frac{1}{2}\right),
$$

hence

$$
\begin{aligned}
S_{N}(f ; y) & =I(f)-\frac{1}{\pi N} \sum_{k=1}^{\infty}(-1)^{k} \cdot \frac{c_{N k}}{k} \cos 2 \pi N k y \\
& +\frac{1}{\pi N} \sum_{k=1}^{\infty}(-1)^{k} \frac{d_{N k}}{k} \sin 2 \pi N k y+\frac{f(0+)-f(1-)}{N} \rho\left(N y+\frac{1}{2}\right) .
\end{aligned}
$$

Setting $y=0$ in (2.18) yields the result of the lemma.

LEMma $2 . r \geqq 2$, integral, $W^{(r)}(x) \geqq \epsilon_{r}>0$ or

$$
\begin{gathered}
W^{(r)}(x) \leqq-\epsilon_{r}<0 \quad \text { for } \quad a \leqq x \leqq b \\
\Rightarrow\left|\int_{a}^{b} \cos W(x) d x\right| \leqq r 2^{(r+1) / 2} \epsilon_{r}^{-1 / r}, \\
\left|\int_{a}^{b} \sin W(x) d x\right| \leqq r 2^{(r+1) / 2} \epsilon_{r}^{-1 / r} .
\end{gathered}
$$


Proof. It is clear that only the inequality $W^{(r)}(x) \geqq \epsilon_{r}>0$ need be considered. The proof only for $\cos W(x)$ will be carried out since that for $\sin W(x)$ is completely similar. The case $r=2$ will be considered first. The function $W^{\prime}(x)$ is monotonic increasing, hence it vanishes at most once in $[a, b]$, say at $x=c$, then

$$
\int_{a}^{b} \cos W(x) d x=\int_{a}^{c} \cos W(x) d x+\int_{c}^{b} \cos W(x) d x .
$$

Let $0 \leqq \delta \leqq b-c$ be chosen, then

$$
\int_{c}^{b} \cos W(x) d x=\int_{c}^{c+\delta} \cos W(x) d x+\int_{c+\delta}^{b} \cos W(x) d x,
$$

and hence

$$
\left|\int_{c}^{b} \cos W(x) d x\right| \leqq \delta+\left|\int_{c+\delta}^{b} \cos W(x) d x\right|
$$

One has

$$
\begin{aligned}
\int_{c+\delta}^{b} \cos W(x) d x & =\int_{c+\delta}^{b} \frac{1}{W^{\prime}(x)} d \sin W(x) \\
& =\frac{1}{W^{\prime}(c+\delta)} \int_{c+\delta}^{\xi} d \sin W(x),
\end{aligned}
$$

in which the second mean-value theorem was used, and hence

$$
\left|\int_{c+\delta}^{b} \cos W(x) d x\right| \leqq \frac{2}{W^{\prime}(c+\delta)} .
$$

Since

$$
W^{\prime}(c+\delta)=\int_{c}^{c+\delta} W^{\prime \prime}(x) d x \geqq \delta \epsilon_{2},
$$

one obtains, from $(2.21)$

$$
\left|\int_{c}^{b} \cos W(x) d x\right| \leqq \delta+\frac{2}{\delta \epsilon_{2}} .
$$

The choice

$$
\delta=\sqrt{2} \epsilon_{2}^{-1 / 2}
$$

yields

$$
\left|\int_{c}^{b} \cos W(x) d x\right| \leqq 2 \sqrt{2} \epsilon_{2}{ }^{-1 / 2} .
$$

The value of $\delta$ in (2.26) may exceed $b-c$, however, in this case the inequality of $(2.27)$ is certainly correct since the integral always admits the estimate $b-c$.

Similarly choose $0 \leqq \delta \leqq c-a$, then

$$
\int_{a}^{c} \cos W(x) d x=\int_{a}^{c-\delta} \cos W(x) d x+\int_{c-\delta}^{c} \cos W(x) d x,
$$


and hence

$$
\left|\int_{a}^{c} \cos W(x) d x\right| \leqq\left|\int_{a}^{c-\delta} \cos W(x) d x\right|+\delta
$$

One has

$$
\begin{aligned}
\int_{a}^{c-\delta} \cos W(x) d x & =\int_{a}^{c-\delta} \frac{1}{W^{\prime}(x)} d \sin W(x) \\
& =\frac{1}{W^{\prime}(c-\delta)} \int_{\xi}^{c-\delta} d \sin W(x)
\end{aligned}
$$

and hence

$$
\left|\int_{a}^{c-\delta} \cos W(x) d x\right| \leqq-\frac{2}{W^{\prime}(c-\delta)}
$$

Since

$$
-W^{\prime}(c-\delta)=\int_{c-\delta}^{c} W^{\prime \prime}(x) d x \geqq \delta \epsilon_{2},
$$

one obtains from $(2.29)$

$$
\left|\int_{a}^{c} \cos W(x) d x\right| \leqq \delta+\frac{2}{\delta \epsilon_{2}}
$$

Hence

$$
\left|\int_{a}^{c} \cos W(x) d x\right| \leqq 2 \sqrt{2} \epsilon_{2}^{-1 / 2}
$$

and, from (2.19),

$$
\left|\int_{a}^{b} \cos W(x) d x\right| \leqq 4 \sqrt{2} \epsilon_{2}^{-1 / 2} .
$$

The lemma is thus established for $r=2$.

Induction will now be employed. The lemma is assumed true for $r=k \geqq 2$. Since $W^{(k+1)}(x)>0, W^{(k)}(x)$ is monotonic increasing, and hence vanishes at most once in $[a, b]$, say at $x=c$. Choose $0 \leqq \delta \leqq b-c$, then

$$
\int_{c}^{b} \cos W(x) d x=\int_{c}^{c+\delta} \cos W(x) d x+\int_{c+\delta}^{b} \cos W(x) d x,
$$

and hence

$$
\left|\int_{c}^{b} \cos W(x) d x\right| \leqq \delta+\left|\int_{c+\delta}^{b} \cos W(x) d x\right| .
$$

The inductive hypothesis states

$$
\left|\int_{c+\delta}^{b} \cos W(x) d x\right| \leqq k 2^{(k+1) / 2} W^{(k)}(c+\delta)^{-1 / k}
$$


hence

$$
\left|\int_{c}^{b} \cos W(x) d x\right| \leqq \delta+k 2^{(k+1) / 2} W^{(k)}(c+\delta)^{-1 / k}
$$

Since

$$
W^{(k)}(c+\delta)=\int_{c}^{c+\delta} W^{(k+1)}(x) d x \geqq \delta \epsilon_{k+1},
$$

one has

$$
\left|\int_{c}^{b} \cos W(x) d x\right| \leqq \delta+k 2^{(k+1) / 2} \delta^{-1 / k} \epsilon_{k+1}^{-1 / k}
$$

The choice

$$
\delta=2^{k / 2} \epsilon_{k+1}^{-1 /(k+1)}
$$

yields

$$
\left|\int_{c}^{b} \cos W(x) d x\right| \leqq(k+1) 2^{k / 2-1 /(k+1)} .
$$

The inequality (2.43) remains correct even for $\delta>b-c$.

Similarly choose $0 \leqq \delta \leqq c-a$, then

$$
\int_{a}^{c} \cos W(x) d x=\int_{a}^{c-\delta} \cos W(x) d x+\int_{c-\delta}^{c} \cos W(x) d x,
$$

and hence

$$
\left|\int_{a}^{c} \cos W(x) d x\right| \leqq\left|\int_{a}^{c-\delta} \cos W(x) d x\right|+\delta .
$$

The inductive hypothesis yields

$$
\left|\int_{a}^{c} \cos W(x) d x\right| \leqq \delta+k 2^{(k+1) / 2}\left[-W^{(k)}(c-\delta)\right]^{-1 / k}
$$

Since

$$
-W^{(k)}(c-\delta)=\int_{c-\delta}^{c} W^{(k+1)}(x) d x \geqq \delta \epsilon_{k+1},
$$

one has

$$
\left|\int_{a}^{c} \cos W(x) d x\right| \leqq \delta+k 2^{(k+1) / 2} \delta^{-1 / k} \epsilon_{k+1}^{-1 / k}
$$

Thus

$$
\left|\int_{a}^{c} \cos W(x) d x\right| \leqq(k+1) 2^{k / 2} \epsilon_{k+1}^{-1 /(k+1)}
$$


and hence

$$
\left|\int_{a}^{b} \cos W(x) d x\right| \leqq(k+1) 2^{(k+2) / 2-1 /(k+1)} \epsilon_{k+1}^{-}
$$

The lemma is now established.

It is now possible to prove

TheOREM 1. $f^{\prime}(x) \in \mathrm{BV}[0,1], L^{(r)}(x) \geqq \epsilon_{r}>0$ for $0 \leqq x \leqq 1$ or

$$
\begin{aligned}
& L^{(r)}(x) \leqq-\epsilon_{r}<0 \quad \text { for } 0 \leqq x \leqq 1, \quad r \geqq 2 \text { integral }, \\
& K_{r}=r 2^{(r+1) / 2-1 / r} \pi^{-1-1 / r} \zeta\left(1+\frac{1}{r}\right) \epsilon_{r}^{-1 / r} \\
\Rightarrow & \left|R_{N}(f)\right| \leqq\left[\left|f^{\prime}(1)\right|+\underset{0}{\mathrm{~V}}\left(f^{\prime}\right)\right] K_{r} N^{-1-1 / r} ;
\end{aligned}
$$

in which $\zeta(x)$ is the Riemann zeta function.

Proof. As in Lemma 1, let

$$
c_{n}=\int_{0}^{1} f^{\prime}(x) \sin [2 \pi n L(x)] d x .
$$

Let

$$
\psi(x)=\int_{0}^{x} \sin [2 \pi n L(u)] d u
$$

then

$$
c_{n}=\int_{0}^{1} f^{\prime}(x) \psi^{\prime}(x) d x
$$

Integrating by parts, one obtains

$$
c_{n}=f^{\prime}(1) \psi(1)-\int_{0}^{1} \psi(x) d f^{\prime}(x) .
$$

Lemma 2 provides the following estimate for $\psi(x)$ :

$$
|\psi(x)| \leqq r 2^{(r+1) / 2-1 / r} \pi^{-1 / r} \epsilon_{r}^{-1 / r} n^{-1 / r},
$$

hence (2.54) and (2.55) yield

$$
\left|c_{n}\right| \leqq\left[\left|f^{\prime}(1)\right|+\mathrm{V}_{0}^{1}\left(f^{\prime}\right)\right] r 2^{(r+1) / 2-1 / r} \pi^{-1 / r} \epsilon_{r}^{-1 / r} n^{-1 / r} .
$$

The infinite series for $R_{N}(f)$ in Lemma 1 may now be estimated. Using (2.56), one obtains

$$
\left|R_{N}(f)\right| \leqq\left[\left|f^{\prime}(1)\right|+\mathrm{V}_{0}^{\mathrm{V}}\left(f^{\prime}\right)\right] r 2^{(r+1) / 2-1 / r} \pi^{-1-1 / r} \epsilon_{r}^{-1 / r} N^{-1-1 / r} \sum_{k=1}^{\infty} k^{-1-1 / r}
$$

Since the infinite series of $(2.57)$ is $\zeta(1+1 / r)$, the inequality of the theorem follows. 
Theorem 2. $f(x)=\int_{-\sigma}^{\sigma} e^{i u x} d \alpha(u), \alpha(u) \in \mathrm{BV}[-\sigma, \sigma]$, $L^{(r)}(x) \geqq \epsilon_{r}>0$ for $0 \leqq x \leqq 1$ or $L^{(r)}(x) \leqq-\epsilon_{r}<0$ for $0 \leqq x \leqq 1$,

$$
\Rightarrow\left|R_{N}(f)\right| \leqq \sqrt{2} \sigma \stackrel{\sigma}{\mathrm{V}}_{-\sigma}(\alpha) K_{r} N^{-1-1 / r} .
$$

Proof. Using the representation

$$
f(x)=\int_{-\sigma}^{\sigma} e^{i u x} d \alpha(u)
$$

one has

$$
f^{\prime}(x)=\int_{-\sigma}^{\sigma} e^{i u x} i u d \alpha(u)
$$

and hence

$$
c_{n}=\int_{-\sigma}^{\sigma} i u\left\{\int_{0}^{1} e^{i u x} \sin [2 \pi n L(x)] d x\right\} d \alpha(u) .
$$

The inner integral of (2.60) may be written

$$
\begin{gathered}
\int_{0}^{1} e^{i u x} \sin [2 \pi n L(x)] d x=\frac{1}{2} \int_{0}^{1} \sin [2 \pi n L(x)-u x] d x \\
+\frac{1}{2} \int_{0}^{1} \sin [2 \pi n L(x)+u x] d x \\
+\frac{1}{2} i \int_{0}^{1} \cos [2 \pi n L(x)-u x] d x-\frac{1}{2} i \int_{0}^{1} \cos [2 \pi n L(x)+u x] d x
\end{gathered}
$$

hence, using Lemma 2 ,

$$
\left|\int_{0}^{1} e^{i u x} \sin [2 \pi n L(x)] d x\right| \leqq r 2^{(r+2) / 2-1 / r} \pi^{-1 / r} \epsilon_{r}^{-1 / r} n^{-1 / r} .
$$

Thus

$$
\begin{aligned}
& \left|c_{n}\right| \leqq r 2^{(r+2) / 2-1 / r} \pi^{-1 / r} \epsilon_{r}^{-1 / r} n^{-1 / r} \int_{-\sigma}^{\sigma}|u||d \alpha(u)|, \\
& \left|c_{n}\right| \leqq \sigma \underset{-\sigma}{\sigma}(\alpha) r 2^{(r+2) / 2-1 / r} \pi^{-1 / r} \epsilon_{r}^{-1 / r} n^{-1 / r} .
\end{aligned}
$$

From the infinite series for $R_{N}(f)$ of Lemma 1, one obtains

$$
\left|R_{N}(f)\right| \leqq \sigma \underset{-\sigma}{\sigma}(\alpha) r 2^{(r+2) / 2-1 / r} \pi^{-1-1 / r} \epsilon_{r}^{-1 / r} N^{-1-1 / r} \sum_{k=1}^{\infty} k^{-1-1 / r},
$$

and hence the result of the theorem.

Theorem 3. $p(x) \equiv 1, f^{\prime}(x) \in \mathrm{BV}[0,1]$

The estimate is sharp.

$$
\Rightarrow\left|R_{N}(f)\right| \leqq \frac{1}{16} \mathrm{~V}_{0}^{1}\left(f^{\prime}\right) N^{-2} .
$$


Proof. Since $p(x) \equiv 1$, one has $L(x)=x$ and hence

$$
c_{n}=\int_{0}^{1} f^{\prime}(x) \sin 2 \pi n x d x .
$$

From (2.11), one has

(2.66) $\frac{1}{N} \int_{0}^{1} \rho\left(N x+\frac{1}{2}\right) f^{\prime}(x) d x=\frac{1}{\pi N} \sum_{k=1}^{\infty} \frac{(-1)^{k}}{k} \int_{0}^{1} f^{\prime}(x) \sin 2 \pi N k x d x$.

After using (2.65) in (2.66) and comparing with the series for $R_{N}(f)$ of Lemma 1 , the following integral expression for $R_{N}(f)$ is obtained.

$$
R_{N}(f)=\frac{1}{N} \int_{0}^{1} \rho\left(N x+\frac{1}{2}\right) f^{\prime}(x) d x .
$$

The Bernoullian function

$$
\sigma(x)=\int_{0}^{x} \rho(u) d u
$$

is periodic with period one and satisfies

$$
0 \leqq \sigma(x) \leqq \frac{1}{8}
$$

In terms of $\sigma(x)$, one has

$$
\begin{aligned}
R_{N}(f) & =-\frac{1}{N^{2}} \int_{0}^{1} \sigma\left(N x+\frac{1}{2}\right) d f^{\prime}(x) \\
& =-\frac{1}{N^{2}} \int_{0}^{1}\left\{\sigma\left(N x+\frac{1}{2}\right)-\frac{1}{16}\right\} d f^{\prime}(x) .
\end{aligned}
$$

Since

$$
\left|\sigma\left(N x+\frac{1}{2}\right)-\frac{1}{16}\right| \leqq \frac{1}{16},
$$

the inequality of the theorem follows. To show that the result is sharp, define $U(x)$ by

$$
\begin{aligned}
U(x) & =1, & & x>0, \\
& =\frac{1}{2}, & & x=0, \\
& =0, & & x<0,
\end{aligned}
$$

and consider functions [1] $f(x)$ for which

$$
f^{\prime}(x)=\operatorname{sgn}\left[1-x-\frac{1}{N} \sum_{j=1}^{N} U\left(\frac{2 j-1}{2 N}-x\right)\right]
$$

then direct computation yields

$$
R_{N}(f)=\frac{1}{4 N}
$$


Since, one readily shows,

$$
{\underset{0}{\mathrm{~V}}}_{\mathrm{V}}^{1}\left(f^{\prime}\right)=4 N
$$

the proof is complete.

3. An Application. In addition to the application of the theorems of this paper to the numerical evaluation of integrals, there are applications of theoretical and design character. One such application is the establishment of approximations to a function $H(t)(-\infty<t<\infty)$ by means of an equal-weight average of the values over $x$ of another function $f(t, x)$. Thus let

$$
H(t)=\int_{0}^{1} p(x) f(t, x) d x
$$

then one has immediately

$$
H(t)=S_{N}(f)+R_{N}(f) .
$$

As an example of (3.2), one may choose

$$
f(t, x)=\cos t x .
$$

Since

$$
\cos t x=\int_{-\sigma}^{\sigma} \cos u x d \alpha(u),
$$

$$
\sigma \geqq t>0
$$

in which $\alpha(u) \in \mathrm{BV}[-\sigma, \sigma]$ and

$$
\begin{aligned}
\alpha(u) & =0, & & u<-t \\
& =\frac{1}{2}, & & -t \leqq u<t, \\
& =1, & & t \leqq u ;
\end{aligned}
$$

one has

$$
{\underset{-\sigma}{\sigma}}_{\mathbf{V}}^{\sigma}(\alpha)=1 .
$$

Thus, a slight modification of Theorem 2 provides the following approximation theorem.

Theorem 4. $H(t)=\int_{0}^{1} p(x) \cos t x d x$

$$
\Rightarrow\left|H(t)-\frac{1}{N} \sum_{j=1}^{N} \cos t x_{j}\right| \leqq K_{r}|t| N^{-1-1 / r}
$$

The same error estimate is obtained if, in place of $\cos t x$, the function $\sin t x$ were used.

Bell Telephone Laboratories, Inc.

Whippany, New Jersey

1. V. I. KRYLov, Approxinate Calculation of Integrals, Fizmatgiz, Moscow, 1959; English transl., Macmillan, New York, 1962, p. 149. MR $22 * 2002$; MR $26 * 2008$. 